OPEN ACCESS

Edited by:

Bruce Campbell,

The University of Melbourne, Australia

Reviewed by:

Leonid Churilov,

The University of Melbourne, Australia

Sami Curtze

University of Helsinki, Finland

${ }^{*}$ Correspondence:

Noreen Kamal

Noreen.Kamal@dal.ca

Specialty section

This article was submitted to

Stroke,

a section of the journal

Frontiers in Neurology

Received: 23 July 2021 Accepted: 30 September 2021 Published: 29 October 2021

Citation:

Bulmer T, Volders D, Blake $J$ and

Kamal N (2021) Discrete-Event

Simulation to Model the Thrombolysis

Process for Acute Ischemic Stroke

Patients at Urban and Rural Hospitals.

Front. Neurol. 12:746404.

doi: 10.3389/fneur.2021.746404

\section{Discrete-Event Simulation to Model the Thrombolysis Process for Acute Ischemic Stroke Patients at Urban and Rural Hospitals}

\author{
Tessa Bulmer ${ }^{1}$, David Volders ${ }^{2,3}$, John Blake ${ }^{1}$ and Noreen Kamal ${ }^{1 *}$ \\ ${ }^{1}$ Department of Industrial Engineering, Faculty of Engineering, Dalhousie University, Halifax, NS, Canada, ${ }^{2}$ Interventional and \\ Diagnostic Neuroradiology, QEIl Health Sciences Centre, Nova Scotia Health, Halifax, NS, Canada, ${ }^{3}$ Department of \\ Radiology, Faculty of Medicine, Dalhousie University, Halifax, NS, Canada
}

Background: Effective treatment with tissue plasminogen activator (tPA) critically relies on rapid treatment. Door-to-needle time (DNT) is a key measure of hospital efficiency linked to patient outcomes. Numerous changes can reduce DNT, but they are difficult to trial and implement. Discrete-event simulation (DES) provides a way to model and determine the impact of process improvements.

Methods: A conceptual framework was developed to illustrate the thrombolysis process; allowing for treatment processes to be replicated using a DES model developed in ARENA. Activity time duration distributions from three sites (one urban and two rural) were used. Five scenarios, three process changes, and two reductions in activity durations, were simulated and tested. Scenarios were tested individually and in combinations. The primary outcome measure is median DNT. The study goal is to determine the largest improvement in DNT at each site.

Results: Administration of tPA in the imaging area resulted in the largest median DNT reduction for Site 1 and Site 2 for individual test scenarios (12.6\%, 95\% Cl 12.4-12.8\%, and $8.2 \%, 95 \% \mathrm{Cl} 7.5-9.0 \%$, respectively). Ensuring that patients arriving via emergency medical services (EMS) remain on the EMS stretcher to imaging resulted in the largest median DNT improvement for Site 3 (9.2\%, 95\% Cl 7.9-10.5\%). Reducing both the treatment decision time and tPA preparation time by $35 \%$ resulted in a $11.0 \%(95 \% \mathrm{Cl}$ $10.0-12.0 \%$ ) maximum reduction in median DNT. The lowest median and $90^{\text {th }}$ percentile DNTs were achieved by combining all test scenarios, with a maximum reduction of $26.7 \%$ (95\% Cl 24.5-28.9\%) and 17.1\% (95\% Cl 12.5-21.7\%), respectively.

Conclusions: The detailed conceptual framework clarifies the intra-hospital logistics of the thrombolysis process. The most significant median DNT improvement at rural hospitals resulted from ensuring patients arriving via EMS remain on the EMS stretcher to imaging, while urban sites benefit more from administering tPA in the imaging area. Reducing the durations of activities on the critical path will provide further DNT improvements. Significant DNT improvements are achievable in urban and rural settings by combining process changes with reducing activity durations.

Keywords: acute ischemic stroke (AIS), door-to-needle time (DNT), tissue plasminogen activator (tPA), thrombolysis, stroke pathways, discrete-event simulation (DES), urban, rural 


\section{INTRODUCTION}

Stroke is a devastating disease, but is treatable with alteplase or tissue plasminogen activator (tPA) (1) and endovascular thrombectomy (EVT) (2-6). Tissue plasminogen activator has been a proven treatment for acute ischemic stroke (AIS) since 1995 (1), and is widely available in urban and rural hospitals. A person having a stroke loses approximately 1.9 million neurons every minute (7), leading to the popular motto "time is brain." Acute ischemic stroke patients should be treated with tPA as rapidly as possible for maximal benefit $(8,9)$, as the effectiveness is highly time dependent (10). Door-to-needle time (DNT) is a critical measure of hospital efficiency linked to patient outcomes and is defined as the time from a patient's hospital arrival to the start of tPA treatment. Fast treatment with tPA has been reported in many urban hospitals, but rural hospitals struggle to reduce treatment times $(11,12)$. A recent study analyzing the thrombolysis process for AIS in urban and rural hospitals highlighted that physician comfort, resource availability, and frequency of treating AIS patients were factors that lead to an inequality in treating patients quickly in rural settings (13). There are key resource differences between urban and rural centers. For instance, rural sites may only have computed tomography (CT) technologists on-call during out of hours, and emergency department (ED) physicians are making the treatment decision, instead of neurologists (13).

Several process changes can reduce DNT $(12,14)$, but these changes are difficult to trial and implement and could disturb established care pathways. Discrete-event simulation (DES) provides a safe and efficient way to model processes and determine the impact of process changes. Operations research techniques, such as simulation, have become a common analytical problem-solving method used in healthcare. Simulation has been amply used to address challenges in ED patient flow optimization (15). Further, simulation allows complex healthcare systems with stochastic elements, such as stroke pathways, to be replicated to provide insights and recommendations for improvements. Discrete-event simulation has been shown to be an effective approach applied to pre-hospital $(16,17)$, intra-hospital $(18-21)$, and both pre and intra-hospital aspects of the acute stroke pathways (2229). Discrete-event simulation literature also spans to areas such as operation of a stroke unit (30), the impact of additional comprehensive stroke centers for EVT (31), and AIS patient disability post-hospital (32). Outcome measures among DES studies include: resource optimization (21), thrombolysis eligibility (16), utilization rate of thrombolysis or intra-arterial thrombectomy $(22,23,27,28)$, and patient outcomes $(22,23,28$, 32 ), amongst others. Improving thrombolysis rates and patient outcomes were the most common aims of the DES studies for acute stroke care found in the literature.

Several studies have illustrated the key activities involved in either the pre-hospital or intra-hospital aspects of acute stroke care $(16,23,24,27-29,33)$. These studies include overviews of intra-hospital thrombolysis steps and also identify different pathway types but lack detail regarding intra-hospital activities and sequences. Additionally, process differences based on pathway type or out of hour resource differences are not well-defined in the literature. We address these gaps by developing a conceptual framework of the intra-hospital aspect of the thrombolysis treatment process by analyzing three urban and rural sites. The framework defines intra-hospital treatment processes, based on patient pathway type, shows resource availability differences in out of hour operations, provides further detail of process activities and sequences, and highlights when potential delays may be encountered. It is important to fill these gaps to provide clarity of intra-hospital logistics, which can lead to solutions to reduce DNT. The study objectives are: 1) to provide a detailed conceptual framework of the thrombolysis process, focusing on intra-hospital activities; and 2) to assess the potential impact of process improvements that can result in faster DNTs when applied to urban and rural settings using a DES model.

\section{METHODS}

A conceptual framework was developed first to provide a generalized model for tasks involved in the thrombolysis process within both urban and rural hospitals. This conceptual model was then used to develop a DES model that can be used for both urban and rural hospitals, based on various inputs. This paper includes descriptions of the conceptual framework, the DES model, as well as the test scenarios applied to the three included sites. The authors declare that all supporting data are available within the article and its Supplementary Material.

\section{Development of Conceptual Framework}

A qualitative study was conducted in Nova Scotia, Canada, to understand the thrombolysis treatment process in urban and rural hospitals. This study provided the foundation for the current research; the full details of this study are published elsewhere (13). There was one urban and two rural sites chosen to enable comparisons between urban and rural hospitals. The respective site distinctions, local target median DNTs, and current median DNTs are detailed in the published qualitative study in the Site Context section (13). The results from that study provided the necessary pathway specifics to create a detailed process map for each site (13), and estimations of activity durations. These process maps were used in this study to develop a conceptual framework of the thrombolysis process in urban and rural hospitals and the DES.

The conceptual framework shows the intra-hospital aspects of the thrombolysis process divided into four panels as shown in Figure 1: (Figure 1A) hospital activities prior to patient arrival; (Figure 1B) arrival activities; (Figure 1C) imaging and treatment decision activities; and (Figure 1D) treatment activities. Figure 1A illustrates the intra-hospital activities that take place during the pre-hospital stage, while the remaining panels represent the hospital-based stage of the treatment process. The framework considers two patient treatment pathways: patients arriving via emergency medical services (EMS), where the stroke protocol has been activated at the hospital prior to the patient's arrival, and patients arriving via 


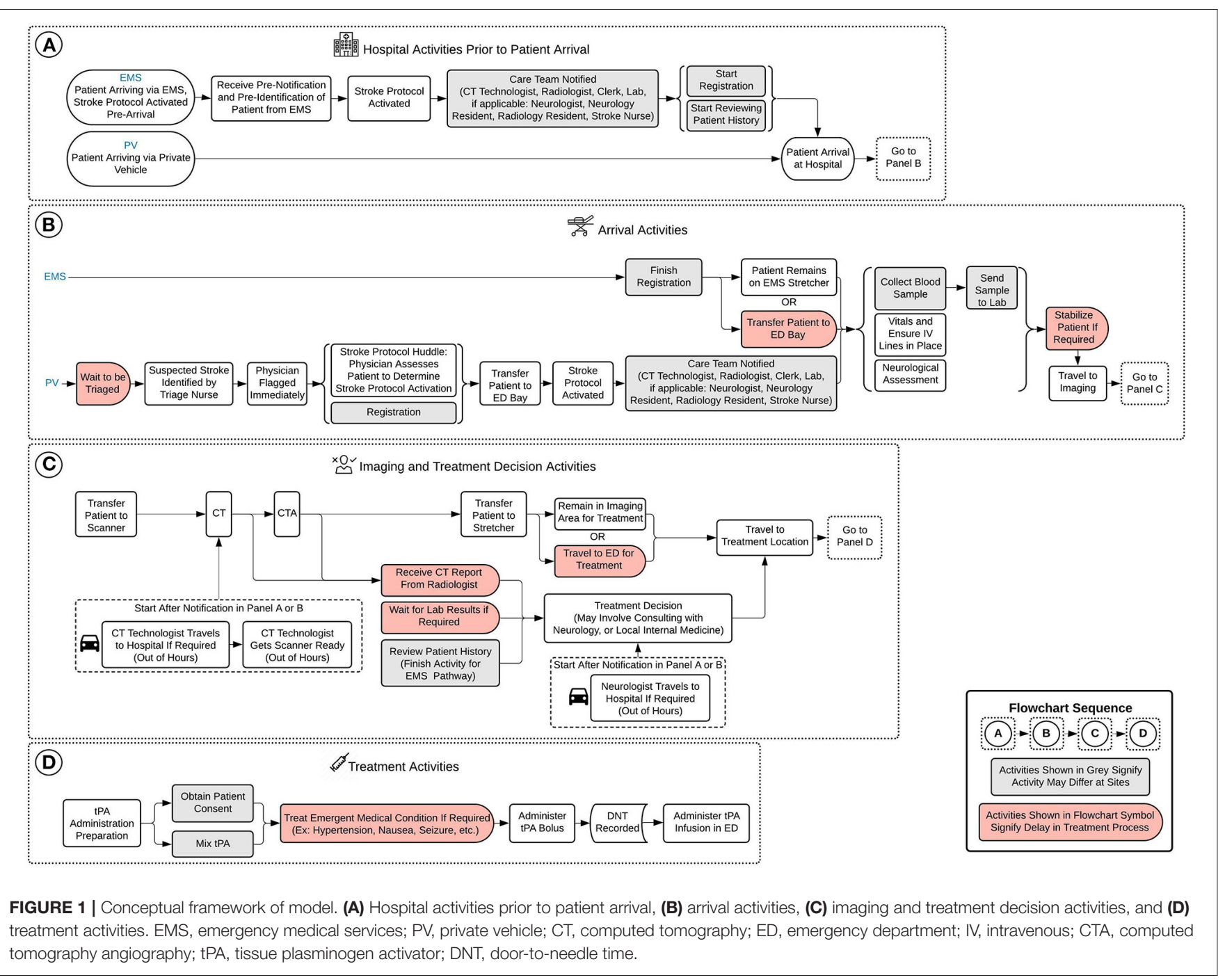

private vehicle (PV). Figures 1A,B display activities in terms of treatment pathways, as the logistics for this portion of the process are pathway dependent. Figure $\mathbf{1 A}$ illustrates efficiencies of the EMS treatment pathway, as several activities can begin in-hospital prior to the patient's arrival. Figure 1B shows the evident difference in activities required to be completed upon arrival between the EMS and PV pathways. When patients enter Figure 1C of the treatment process, imaging and treatment decision activities, the process is identical for both patients arriving via EMS and PV. Activities shown in gray in Figure 1 signify the activity may differ by site regarding how and when the activity is executed, as the healthcare professionals responsible for completing the activities may differ. Activities shown in red in Figure 1 signify a potential delay in the treatment process. While patient-related delays cannot be entirely avoided, they can be minimized with appropriate anticipation and preparation. However, system delays (i.e., delays not specifically related to the patient) can, and should, be eliminated to achieve more streamlined treatment.

\section{Simulation Model}

A DES model was developed based on the conceptual framework shown in Figure $\mathbf{1}$ and the previously developed process maps (13), and applied to the three sites to verify the model and provide context for the test scenarios. The model provides representation of the intra-hospital aspect of the thrombolysis process for urban and rural hospitals. The model includes the two acute stroke pathways noted in Figure 1. The strengthening the reporting of empirical simulation studies guidelines developed for discrete-event simulations (STRESS-DES) was consulted to report key aspects of the DES model in the following domains: objectives, logic, data, experimentation, implementation, and code access (34).

\section{Objectives}

The DES aims to replicate current practice of the thrombolysis treatment process of AIS patients for each site and gives the ability to assess the potential impact of process improvements that can result in reduced DNT. 
TABLE 1 | Test scenarios and current site processes.

\begin{tabular}{|c|c|c|c|}
\hline \multirow[t]{2}{*}{ Test scenarios } & \multicolumn{3}{|c|}{ Is the test scenario already implemented at the site? (i.e., included in site baseline) } \\
\hline & Site 1 (Urban) & Site 2 (Rural) & Site 3 (Rural) \\
\hline (P1) Patients arriving via EMS remain on EMS stretcher to imaging & Yes & Yes & No (patient transferred to ED bay) \\
\hline (P2) Administration of tPA in imaging area (regular and out of hours) & No (during regular hours only) & No (administered in ED) & No (administered in ED) \\
\hline (P3) Pre-registration of patients arriving via EMS & Yes & Yes & No (completed upon arrival) \\
\hline (R1) Reduce treatment decision time by $35 \%$ & - & - & - \\
\hline (R2) Reduce tPA administration preparation time by $35 \%$ & - & - & - \\
\hline
\end{tabular}

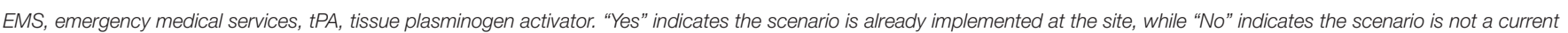
site process and the current site process is subsequently defined.

\section{Test Scenarios}

In addition to current baseline treatment processes, five scenarios were tested for each site. The scenarios are three process changes and two reductions in activity durations. Process change scenarios entail implementing a change in a site's current thrombolysis treatment process, while reduction in activity duration scenarios reduces the amount of time taken to execute a specified activity. The five scenarios are defined in Table 1, along with defining current site processes. The process changes were chosen because they are well-supported in previous studies to have reduced DNTs. Activities chosen to test the effect of a reduction in duration were selected where there is considerable variability in activity length of time, and evidence that times can be reduced with standardized protocols. The scenarios were tested individually, and in combinations.

The following lists and details the five test scenarios that were run and applied to the three included sites in this study:

Process change 1 (P1): Patients arriving via EMS remain on EMS stretcher to imaging $(12,14,35-39)$ - This scenario models the impact of keeping a patient arriving via EMS on the EMS stretcher, as opposed to transferring the patient to an ED bay to travel to imaging.

Process change 2 (P2): Administration of tPA in imaging area (regular and out of hours) $(14,35,36,40-42)$-This scenario models the administration of the tPA bolus in the imaging area, as opposed to administration in the ED following imaging. This scenario eliminates the travel time from imaging to the ED prior to the administration of the bolus and has a reduced tPA administration preparation activity time.

Process change 3 (P3): Pre-registration of patients arriving via EMS (14, 40, 41)-This scenario models the pre-registration of patients traveling via the EMS pathways, as opposed to starting the registration process when the patient arrives at the hospital.

Reduction in activity duration 1 (R1): Reduce treatment decision time by $35 \%$ - This scenario models a reduction in the time taken for the physician to decide whether the patient will receive thrombolysis treatment by $35 \%$.

Reduction in activity duration 2 (R2): Reduce tPA administration preparation time by $35 \%$-This scenario models a reduction in the time taken to complete the preparation required before administering tPA by 35\%. Tissue plasminogen activator administration preparation encompasses activities such as: obtaining the drug, estimating the patient's weight, calculating the correct dosing, mixing the tPA, and programming the tPA administration pump.

\section{Outcome Measures}

The primary outcome measure for this study was median DNT for patients treated with thrombolysis, and the measure of variance for this outcome was interquartile range (IQR). The reduction in IQR was also reported. A critical outcomes measure that was included was $90^{\text {th }}$ percentile DNT for patients treated with thrombolysis. This measure is important as it shows the DNT where most ( $90 \%$ of patients) are treated within, and there has been previous justification for the use of this measure in acute stroke processes (43). The current Canadian Guidelines state that the $90^{\text {th }}$ percentile for DNT should be $60 \mathrm{~min}(44)$.

\section{Statistical Analysis}

The Mann-Whitney U-test and the Chi-Square Goodness-of-Fit test were performed for continuous and categorical variables, respectively. Minitab Statistical Software for Windows (Minitab, State College, PA, version 19) was used for all statistical analysis. A $p$-value of $<0.05$ was considered statistically significant.

\section{Logic}

The model considers variation in patient pathway and activity durations using probability distributions and employs a stochastic patient arrival rate schedule. Once patients arrive, the model distributes patient pathways with approximately $80 \%$ of patients arriving via EMS, and $20 \%$ arriving via PV. Activity duration distributions are based on estimations from healthcare professionals at the included sites. A time study would be required to verify the activity duration distributions. The modeled process baseline median DNTs matched the sites' current median DNTs, which provided some verification of the estimates. The arrival rate schedule was approximated from Site 1 data to more accurately replicate hourly volumes in which AIS patients arrive at a hospital. As aggregate data showed that Site 2 and Site 3 had lower ischemic stroke volumes compared to Site 1 (with Site 3 encountering the least amount of AIS patients), their arrival rates were adjusted accordingly. The time and day of a patient's arrival in the system can impact their treatment pathway due to resource availability. Regular hours are defined as 8:00 a.m. to 4:00 p.m., Mondays to Fridays, while out of hours are considered all times outside of regular 
TABLE 2 | Site 1 model verification of arrival rate and pathway types.

\begin{tabular}{|c|c|c|c|c|c|c|}
\hline Time period & Desired rate & Model rate & Desired proportion & Desired volume & Model volume & $p$-Value \\
\hline 12:00 a.m. $-2: 00$ a.m. & 0.00428 & 0.01308 & 0.0310 & 93.7 & 79 & 0.169 \\
\hline 2:00 a.m.-4:00 a.m. & 0.00321 & 0.01291 & 0.0233 & 70.4 & 78 & \\
\hline 4:00 a.m.-6:00 a.m. & 0.00321 & 0.01274 & 0.0233 & 70.4 & 77 & \\
\hline 6:00 a.m.-8:00 a.m. & 0.00642 & 0.02416 & 0.0465 & 140.5 & 146 & \\
\hline 8:00 a.m.-10:00 a.m. & 0.01391 & 0.05660 & 0.1008 & 304.5 & 342 & \\
\hline 10:00 a.m.-12:00 p.m. & 0.01819 & 0.06207 & 0.1318 & 398.2 & 375 & \\
\hline 12:00 p.m.-2:00 p.m. & 0.01926 & 0.06869 & 0.1395 & 421.4 & 415 & \\
\hline 2:00 p.m.-4:00 p.m. & 0.01712 & 0.05776 & 0.1240 & 374.6 & 349 & \\
\hline 4:00 p.m.-6:00 p.m. & 0.01712 & 0.06223 & 0.1240 & 374.6 & 376 & \\
\hline 6:00 p.m.-8:00 p.m. & 0.01605 & 0.05925 & 0.1163 & 351.3 & 358 & \\
\hline 8:00 p.m.-10:00 p.m. & 0.01070 & 0.03608 & 0.0775 & 234.1 & 218 & \\
\hline 10:00 p.m.-12:00 a.m. & 0.00856 & 0.03443 & 0.0620 & 187.3 & 208 & \\
\hline Definition of time period & \multicolumn{3}{|c|}{ Desired percentage of arrivals (\%) } & \multicolumn{3}{|c|}{ Model output (\%) } \\
\hline Regular hours (Monday-Friday, 8:00 a.m.-4:00 p.m.) & \multicolumn{3}{|c|}{35.4} & \multicolumn{3}{|c|}{34.5} \\
\hline $\begin{array}{l}\text { Out of hours (Monday-Friday, 4:00 p.m.-8:00 a.m., } \\
\text { Saturday and Sunday) }\end{array}$ & \multicolumn{3}{|c|}{64.6} & \multicolumn{3}{|c|}{65.5} \\
\hline Pathway type & \multicolumn{3}{|c|}{ Desired pathway distribution (\%) } & \multicolumn{3}{|c|}{ Model output (\%) } \\
\hline EMS & \multicolumn{3}{|c|}{80.0} & \multicolumn{3}{|c|}{79.5} \\
\hline PV & \multicolumn{3}{|c|}{20.0} & \multicolumn{3}{|c|}{20.5} \\
\hline
\end{tabular}

EMS, emergency medical services; PV, private vehicle. Analysis was performed using the chi-square goodness-of-fit test.

hours. The schedule generates approximately $35 \%$ of arrivals in regular hours and $65 \%$ during out of hours as determined from our input data. The schedule specifies that the majority of arrivals occur between 8:00 a.m. and 8:00 p.m., with few arrivals between 12:00 a.m. and 8:00 a.m. Human resource and the resulting treatment process differences between regular and out of hours are defined for each site in the Section 2 Treatment Process Results section of the qualitative study (13). In addition to the conceptual framework shown in Figure 1, ARENA simulation images (Supplementary Figures 1-6) and model activity duration distributions for process baselines and all test scenarios (Supplementary Table 1) can be found in Supplementary Material for further clarity.

\section{Data}

The model activity duration distributions (Supplementary Table 1) and arrival rate schedules (Supplementary Table 2) are detailed in Supplementary Material. Additionally, verification techniques (Supplementary Tables 3-5), sensitivity analysis results (Supplementary Table 6), and number of replications analysis results (Supplementary Table 7) are detailed in Supplementary Material. Arrival rate and pathway type verification results are shown in Table 2.

The DES has several assumptions and simplifications:

1. Due to the top prioritization of acute stroke patients within a hospital, it is assumed these patients will receive priority for the required resources upon arrival, and that the resources are always available.
2. Patient pathway type assignments (EMS or PV) are independent.

3. The EMS pathway always provides the hospital with prenotification and pre-identification.

4. Activity durations are independent of each other.

5. All patients in the DES model are eligible to receive thrombolysis treatment.

6. The system may have only a single stroke protocol activation at a time.

7. The model assumes $100 \%$ stroke protocol compliance and does not consider personnel dependent variation. Although, it should be noted that waiting for a patient's lab results is not always required to determine the thrombolysis treatment decision, which is reflected in the duration distribution for this activity.

There are various reasons based on literature for the established DES assumptions that allow the model to produce meaningful results. Assumption 1 was established due to the Canadian triage and acuity scale (CTAS) level assigned to AIS patients in the qualitative study which provided the foundation for this work (13). Assumptions 2 and 4 are in agreement with a common DES model assumption made regarding acute stroke systems, which is independence of patient attributes (33). Assumptions 3 and 6 are considered appropriate based on expert-opinion from interview participants in the founding qualitative study (13) that indicated pre-identification and pre-notification are generally received for patients traveling via the EMS pathway, and that it would be a rare occurrence to have more than one stroke protocol activation simultaneously at a hospital. As this study focuses on the impact of process improvements on DNT, assumption 
TABLE 3 | Median DNT results by scenario for Site 1, Site 2, and Site 3.

\begin{tabular}{|c|c|c|c|c|c|c|c|c|c|}
\hline & \multicolumn{3}{|c|}{ Site 1 (Urban) } & \multicolumn{3}{|c|}{ Site 2 (Rural) } & \multicolumn{3}{|c|}{ Site 3 (Rural) } \\
\hline & $n$ & Median DNT (IQR) (min) & $p$-Value & $n$ & Median DNT (IQR) (min) & $p$-Value & $n$ & Median DNT (IQR) (min) & $p$-Value \\
\hline Process baseline & 3,021 & $50.0(45.4-53.8)$ & - & 780 & $40.1(38.7-48.0)$ & - & 405 & $74.0(70.8-82.6)$ & - \\
\hline \multicolumn{10}{|l|}{ Process changes } \\
\hline P1 & 3,021 & Current baseline & - & 780 & Current baseline & - & 405 & $66.4(63.6-75.7)$ & $<0.0001$ \\
\hline P2 & 2,984 & 43.7 (42.2-46.3) & $<0.0001$ & 856 & 36.6 (34.9-44.6) & $<0.0001$ & 405 & $71.0(66.0-80.3)$ & $<0.0001$ \\
\hline P3 & 3,021 & Current baseline & - & 780 & Current baseline & - & 405 & 71.9 (68.6-80.9) & $<0.0001$ \\
\hline \multicolumn{10}{|c|}{ Reduction in activity durations } \\
\hline $\mathrm{R} 1$ & 3,021 & $50.0(45.4-53.8)$ & 1.000 & 780 & $39.8(38.5-48.0)$ & 0.1430 & 405 & $72.3(70.0-76.0)$ & $<0.0001$ \\
\hline $\mathrm{R} 2$ & 3,022 & $45.7(42.0-49.1)$ & $<0.0001$ & 780 & 37.8 (35.9-48.0) & $<0.0001$ & 405 & $71.8(66.6-81.4)$ & $<0.0001$ \\
\hline \multicolumn{10}{|c|}{ Combinations of process changes and reduction in activity durations } \\
\hline P1, P2, P3 & 2,984 & $43.7(42.2-46.3)$ & $<0.0001$ & 856 & $36.6(34.9-44.6)$ & $<0.0001$ & 405 & $61.3(56.3-72.6)$ & $<0.0001$ \\
\hline $\mathrm{R} 1, \mathrm{R} 2$ & 3,022 & $45.7(42.0-49.1)$ & $<0.0001$ & 780 & $36.7(35.4-48.0)$ & $<0.0001$ & 405 & $65.3(62.8-70.6)$ & $<0.0001$ \\
\hline P1, P2, P3, R1, R2 & 2,984 & 40.6 (39.1-43.2) & $<0.0001$ & 856 & 33.6 (32.4-44.6) & $<0.0001$ & 405 & $52.9(49.8-61.2)$ & $<0.0001$ \\
\hline
\end{tabular}

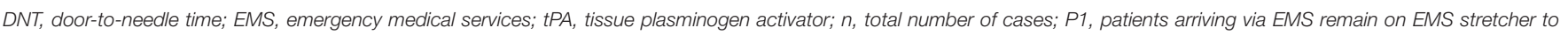

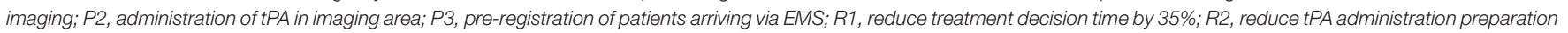
time by $35 \%$. Analysis was performed using the Mann-Whitney U-test comparing DNT data from the changes that were implemented to the respective site's baseline DNT data.

5 ensured all patients included in the model received tPA thus outputting a DNT. The final assumption 7 was established due to a lack of data regarding compliance variation, as well as allowing the maximum potential of a process improvement to be illustrated.

The following are model simplifications:

1. Activity durations are independent of the onset to arrival time, meaning durations do not change if a patient is approaching the 4.5 -h window.

2. Only AIS patients are included; stroke mimics, and hemorrhagic stroke patients are excluded.

3. Ineligible thrombolysis candidates are not considered.

4. Stroke severity, patient age, and patient sex are not considered.

Certain variation in activities may be due to factors included in the assumptions and simplifications listed above; for example, milder stroke patients often have longer treatment times, due to the time taken to determine a diagnosis, and treatment decision. These variations in the associated activities are considered in the distribution of each relevant activity in the DES.

\section{Experimentation}

The model did not include a warm-up period due to the transient nature of the treatment process being modeled. As randomness exists in all simulation results, a number of replications analysis was conducted to determine the number of runs required to achieve a particular precision of the outcome estimate, summarized in Supplementary Table 7 in Supplementary Material. It was determined that model runs would consist of 30 replications. As real site median DTN data from Nova Scotia Health was provided for a period of 1 year, a replication length of 1 year was chosen for the model.

\section{Implementation}

The simulation model was developed using ARENA software (Rockwell Automation, Milwaukee, WI, version 16.00.00003).

\section{Code Access}

To obtain access to the developed ARENA model, please contact the corresponding author.

\section{Ethics}

Ethics approval was obtained from the Nova Scotia Health Research Ethics Board (REB) for this study, with the REB file number 1025975.

\section{RESULTS}

The model was used to determine the impact of process changes and reduction in activity durations on DNT at each site in comparison to the site-specific baseline DNT. The actual median DNT for Site 1, Site 2, and Site 3 are 50.0, 40.0 and $77.5 \mathrm{~min}$ (June 2019-May 2020), with modeled baseline median DNT of 50.0 (IQR 45.4-53.8), 40.1 (IQR 38.7-48.0), and 74.0 (IQR 70.8$82.6) \mathrm{min}$, respectively. The results of the model baselines and test scenarios are summarized in Table 3, with the median DNT and IQR for the total 30 replications calculated for each scenario.

\section{Test Scenario Experiment Results}

The results of all scenario experiments are described below and summarized in Tables 3, 4 and Figures 2, 3. Table 3 includes median DNT and IQR range results for each scenario experiment, and the total number of cases used in the calculations for median DNT for each scenario.

Figures 2, 3 illustrate DNT reduction percentage, IQR reduction percentage, and 90th percentile DNT reduction percentage results for all three sites for all individual test scenarios, and combinations of scenarios, respectively. The $90^{\text {th }}$ 
TABLE 4 | $90^{\text {th }}$ percentile DNT results by scenario for Site 1, Site 2, and Site 3.

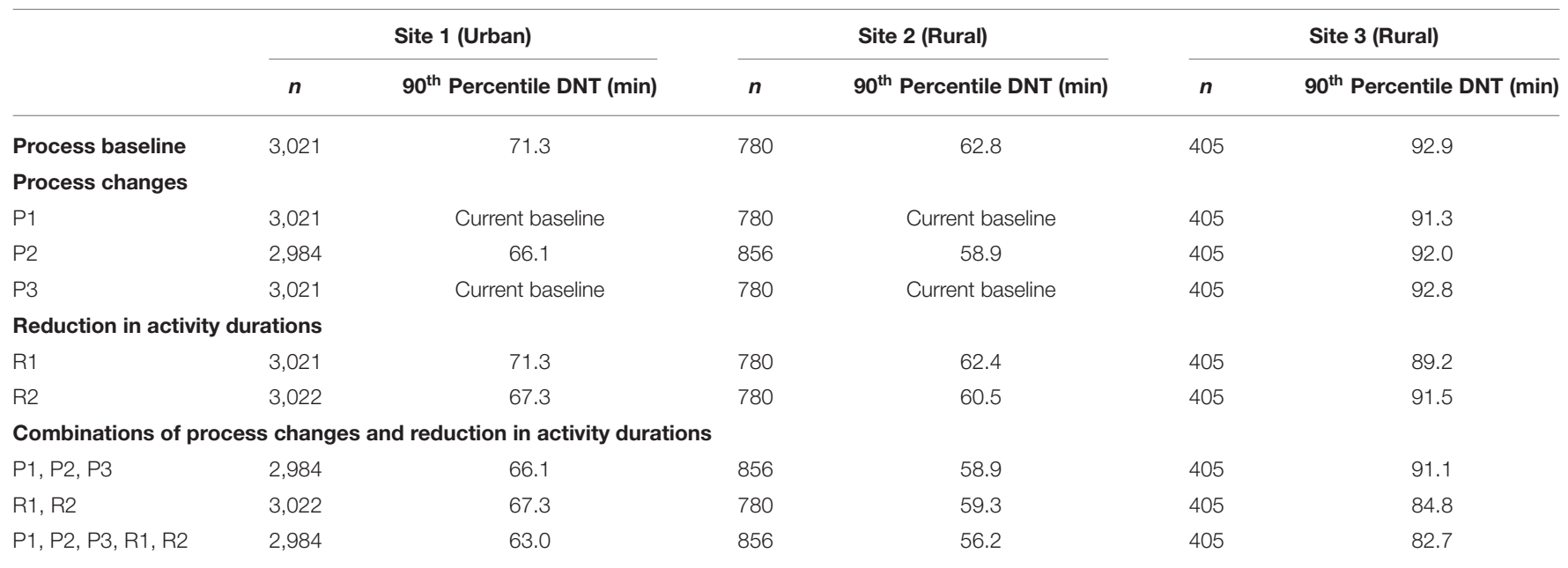

DNT, door-to-needle time; EMS, emergency medical services; tPA, tissue plasminogen activator; $n$, total number of cases; $P 1$, patients arriving via EMS remain on EMS stretcher to

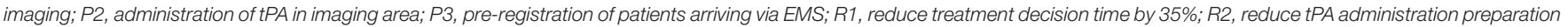
time by $35 \%$.
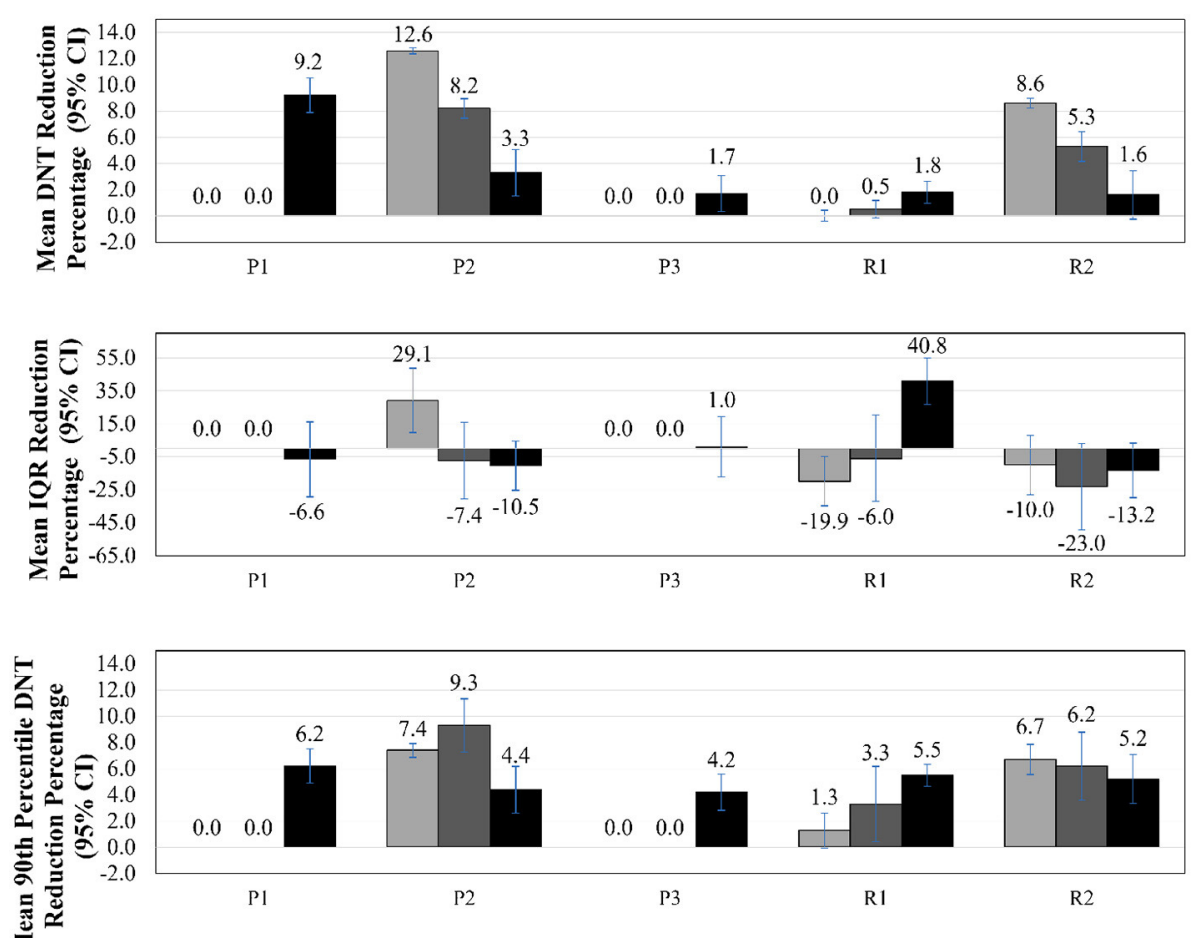

Individual Test Scenarios

$\square$ Site 1 (Urban) घSite 2 (Rural) घSite 3 (Rural)

FIGURE 2 | DNT reduction, IQR reduction, 90 ${ }^{\text {th }}$ percentile DNT reduction-individual test scenario results. DNT, door-to-needle time; Cl, confidence interval; IQR, interquartile range; $\mathrm{P} 1$, patients arriving via EMS remain on EMS stretcher to imaging; P2, administration of tPA in imaging area; $\mathrm{P} 3$, pre-registration of patients arriving via EMS; R1, reduce treatment decision time by 35\%; R2, reduce tPA administration preparation time by $35 \%$.

percentile DNT results are summarized in Table 4. Note that process changes ( $\mathrm{P} 1, \mathrm{P} 2$, or $\mathrm{P} 3)$ tested that resulted in DNT reductions of zero signify the site was already implementing that scenario as part of their baseline.
Patients Arriving via EMS Remain on EMS Stretcher to Imaging (P1)-As Site 1 and Site 2 were currently implementing this process as their baseline, those sites did not experience an improvement in DNT. This process change did benefit Site 3 , and 

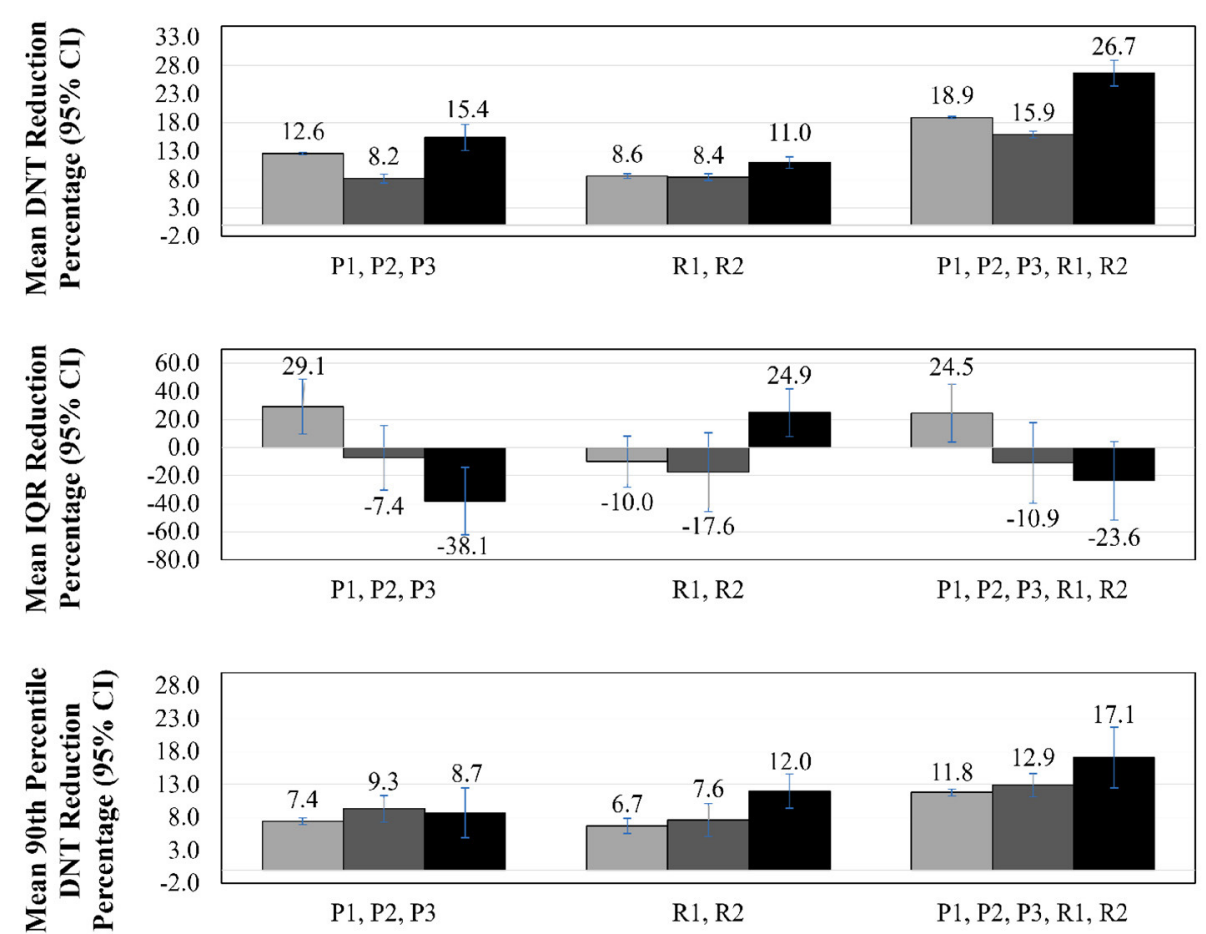

Test Scenario Combinations

uSite 1 (Urban) घ Site 2 (Rural) \Site 3 (Rural)

FIGURE 3 | DNT reduction, IQR reduction, 90 ${ }^{\text {th }}$ percentile DNT reduction-test scenario combinations results. DNT, door-to-needle time; CI, confidence interval; IQR, interquartile range; $\mathrm{P} 1$, patients arriving via EMS remain on EMS stretcher to imaging; P2, administration of tPA in imaging area; $\mathrm{P} 3$, pre-registration of patients arriving via EMS; R1, reduce treatment decision time by 35\%; R2, reduce tPA administration preparation time by $35 \%$.

led to the largest improvement for Site 3 across all individual test scenarios. The DNT at Site 3 was reduced to 66.4 (IQR 63.6-75.7) min from a median of 74.0 (IQR 70.8-82.6) min, translating to a 9.2\% (95\% CI 7.9-10.5\%) improvement in DNT compared to the respective site baseline.

Administration of tPA in Imaging Area (Regular and Out of Hours) (P2)_Administration of tPA to patients in the imaging area resulted in a reduction of the median DNT for Site 1, Site 2, and Site 3 to 43.7 (IQR 42.2-46.3) $\mathrm{min}, 36.6$ (IQR 34.9-44.6) min, and 71.0 (IQR 66.0-80.3) min, respectively. This scenario was the most impactful individual test result for Site 1 and Site 2, leading to a reduction of $12.6 \%$ (95\% CI 12.4-12.8\%) and 8.2\% (95\% CI 7.5-9.0\%), respectively. It was anticipated that Site 1 would show the largest improvement for this scenario, as Site 1 has the longest travel time to the ED from imaging since it is large tertiary care center.

Pre-registration of Patients Arriving via EMS (P3)-As Site 1 and Site 2 include this process in their baseline, those sites did not experience an improvement in DNT. This process change resulted in a DNT of 71.9 (IQR 68.6-80.9) min and a reduction of $1.7 \%$ (95\% CI $0.3-3.1 \%)$ at Site 3.

Reduce Treatment Decision Time by 35\% (R1)_Physician comfort with treatment with tPA results in a shorter amount of time for making the treatment decision. The reduction in decision time did not reduce Site 1's DNT, highlighting that a bottleneck was present elsewhere. Scenario R1 also had little impact on Site 2, with a $0.5 \%$ (95\% CI -0.1-1.2\%) improvement in DNT. Site 3 experienced a $1.8 \%$ (95\% CI 1.02.7\%) improvement, with a DNT of 72.3 (IQR 70.0-76.0) min.

Reduce tPA Administration Preparation Time by 35\% (R2)This reduction in activity duration scenario benefitted Site 1 and Site 2 to a greater degree than did R1. The DNT was reduced for Site 1 by $8.6 \%$ (95\% CI 8.2-9.0\%) with a DNT of 45.7 (IQR 42.049.1 ) min. Site 2 improved their DNT by $5.3 \%$ (95\% CI $4.2-6.5 \%$ ) with an output of 37.8 (IQR 35.9-48.0) min. The impact of R2 was comparable to R1 for Site 3, with DNT reduction of $1.6 \%$ (95\% CI $-0.2-3.5 \%$ ) and a DNT of 71.8 (IQR 66.6-81.4) $\mathrm{min}$.

Combination of P1, P2, P3-When combining all process change scenarios, Site 3 saw a $15.4 \%$ (95\% CI $13.1-17.7 \%)$ reduction with a DNT of 61.3 (IQR 56.3-72.6) min from a baseline median of 74.0 (IQR 70.8-82.6) $\mathrm{min}$. As Site 1 and Site 2 were currently implementing $\mathrm{P} 1$ and $\mathrm{P} 3$, their respective results are identical to their $\mathrm{P} 2$ results as that was the only new process change for those sites.

Combination of $R 1, R 2$-Combining the two reductions of activity duration scenarios benefited Site 3 far more than Site 1 and Site 2, whose improvements in this case were largely attributable to R2. The combination of R1 and R2 was equally, or more, effective in terms of improving DNT when compared to being individually tested, due to the close relationship of these 
activities and the bottleneck formed when adjusted individually. The resulting DNT for Site 1, Site 2, and Site 3 were 45.7 (IQR 42.0- 49.1) min, 36.7 (IQR 35.4-48.0) $\mathrm{min}$, and 65.3 (IQR 62.8-70.6) min, respectively.

Combination of P1, P2, P3, R1, R2-As anticipated, the lowest DNT for each site was achieved by combining all test scenarios, which resulted in the following DNTs: Site 1 was reduced by 18.9\% (95\% CI 18.6-19.1\%) to 40.6 (IQR 39.1-43.2) min, Site 2 by $15.9 \%$ (95\% CI $15.3-16.4 \%$ ) to 33.6 (IQR 32.4-44.6) min, and Site 3 by $26.7 \%$ (95\% CI 24.5-28.9) to 52.9 (IQR 49.8-61.2) min.

In addition to median DNT and DNT reduction percentages results, Figures 2, 3 illustrate IQR reduction percentages and $90^{\text {th }}$ percentile DNT reduction percentages results which further define the spread of the DNT results. As shown in Figures 2, 3, Site 2 did not experience a reduction in IQR with individual or combinations of test scenarios. It can be seen that the following individual test scenarios led to the largest reduction in IQR for Site 1 and Site 3, respectively: P2 with a reduction in IQR of $29.1 \%$ (95\% CI 9.7-48.5\%), and R1 with a reduction in IQR of $40.8 \%$ (95\% CI 26.7-54.8\%). Site 1 experienced a reduction in IQR in all test scenarios combinations involving $\mathrm{P} 2$, such as: P1, P2, P3 (reduction of $29.1 \%, 95 \%$ CI 9.7-48.5\%), and P1, P2, P3, R1, R2 (IQR reduction of $24.5 \%, 95 \%$ CI $4.0-45.0 \%$ ). Site 3 also had a reduced IQR for test scenario combination R1, R2 (IQR reduction of $24.9 \%, 95 \%$ CI $7.9-42.0 \%$ ). The majority of individual and combinations of test scenarios resulted in an increased IQR. The lowest $90^{\text {th }}$ percentile DNT experienced in individual test scenarios for Site 1, Site 2 and Site 3, respectively, as shown in Table 4, are the following: $66.1 \mathrm{~min}$ resulting from P2 (reduction of 7.4\%, 95\% CI 6.9-8.0\%), 58.9 min resulting from P2 (reduction of 9.3\%, 95\% CI 7.3-11.3\%), and 89.2 min resulting from R1 (reduction of 5.5\%, 95\% CI 3.0-7.9\%). The lowest $90^{\text {th }}$ percentile DNT results when considering all individual and combinations of test scenarios were achieved by combining all three process changes and two reduction in activity durations for all sites. When combining all test scenarios, the following $90^{\text {th }}$ percentile DNT improvements were experienced: Site 1 was reduced from 71.3 to $63.0 \mathrm{~min}$ (reduction of $11.8 \%, 95 \%$ CI 11.3$12.3 \%$ ), Site 2 was reduced from 62.8 to 56.2 min (reduction of $12.9 \%, 95 \%$ CI 11.2-14.7\%), and Site 3 was reduced from 92.9 to 82.7 min (reduction of $17.1 \%, 95 \%$ CI $12.5-21.7 \%$ ).

\section{DISCUSSION}

The developed conceptual framework expands upon published works (33) to include details extending past key treatment activities, and highlights when delays may be encountered. The framework can be used to promote discussion among healthcare professionals treating AIS patients by facilitating brainstorming methods to streamline processes. It is important when creating a framework to represent urban and rural settings, to accommodate for treatment process differences between them. Low volumes of thrombolysis treatment in rural hospitals makes it challenging to trial process changes, making DES a beneficial tool to incorporate process improvement. A recent study that reduced DNTs across an entire population further illustrated the challenge in improving DNT in rural settings as rural hospitals were only able to reduce their DNTs to a median of $54 \mathrm{~min}$ from $84 \mathrm{~min}$ after the year-long study (11). Discreteevent simulation modeling can provide hospitals with an initial assessment of treatment changes to then implement those that will have the largest impact, without needing to commit resources for trial. Using a DES model allows the potential impact of process improvements to be quantified, providing an initial assessment pre-implementation, and evidence for further trial. The included case studies provided context for the results, demonstrating the impact of treatment changes in a group of hospitals with varying characteristics which allows the findings from the developed model to be applicable elsewhere with no additional work required. Although, the model could be modified for further assessment of treatment changes at additional sites.

The study shows that the urban and rural sites can benefit from process improvements, by implementing process changes or reducing activity durations. As anticipated, the applied test scenarios resulted in a larger DNT reduction in Site 3, as it was not implementing any of the scenarios and had the longest median DNT. It should be noted that Site 3's DNT is capable of being lower than this study has illustrated, if the reduction in activity duration scenarios reduced durations to a set value, vs. reducing the site's current durations by $35 \%$. The individual and combination testing of the reduction in activity duration scenarios illustrated that reducing one activity duration can create a bottleneck elsewhere, and in situations with parallel activities, all activities on the critical path must be reduced to see an impact. In addition to focusing on the impact of treatment changes on median DNT, it is important to also acknowledge the $90^{\text {th }}$ percentile DNT (43). The $90^{\text {th }}$ percentile DNT metric defines the timeframe in which the majority of patients are treated, and according to the current Canadian Guidelines the $90^{\text {th }}$ percentile DNT should be $60 \mathrm{~min}$ (44). The study results show that Site 2 can achieve this standard with minimal process improvements, while Site 1 is close with the implementation of all test scenarios.

The DNT improvements from the test scenarios using the DES model resulted in less reduction than reported in the literature, where reductions of $20-40 \%$ have resulted from implementing similar process changes $(11,12,35$, $37,38,40)$. There are many reasons for this, as the implementation of a process change may result in activity duration reductions across the entire treatment process. The magnitude of DNT improvements shown in the literature for implemented changes, not simulated, may be attributable to a behavioral component of implementation, meaning greater reductions in DNT may be realized through implementation. Finding solutions using DES modeling that result in reduced DNTs has the potential to improve clinical outcomes due to the established relationship between treatment times and patient outcomes $(8,9)$. Additional hospital-based strategies such as: single-call activation of care team, rapid acquisition of brain imaging, point of care International Normalized Ratio 
(INR) testing, rapid access to tPA, and not requiring written informed consent $(14,35,40)$, should be incorporated for further improvements.

The benefit of trialing process changes within a DES model is clear, although there are challenges involved in developing an appropriately accurate model to represent the system. A study focusing on the development of hyperacute stroke care frameworks summarized the main two difficulties concisely: (1) modelers must understand clinical concepts and have the ability to grasp the pathway logistics; and (2) translate that knowledge into a model for use of testing (33). This highlights the importance of hyperacute stroke frameworks and the need for providing as much detail as possible for logistics to be understood thoroughly. Only including key activities in these frameworks and models may limit the extent of the improvement. Additionally, access to the required data, including activity durations, to establish a reasonable representation of the system is a challenge. To have an accurate description of activity durations, data collection studies, such as time studies, would be beneficial. Time studies are useful in providing required data in areas such as: activity durations based on triage category (45), and clinician work time allocation and interruptions (46), enabling further model accuracy. It is advantageous for healthcare professionals treating AIS patients to be aware of this data, as awareness of benchmarks could lead to DNT improvements with a team culture to provide rapid care and establish a continuous improvement mindset.

\section{Limitations}

A limitation of this study is that the conceptual framework was based on only three hospitals with a small sample size of participants involved at each site in a single Canadian province (13). Furthermore, only two rural sites were studied, meaning the conceptual framework presented may not address all urban-rural treatment differences. It would be advisable to incorporate additional urban and rural hospitals to ensure variations in treatment are encompassed fully. As the conceptual framework was based on interviews conducted in a single Canadian province, the framework likely includes inefficiencies in their system. The process can have additional efficiencies by implementing the following: completing the neurological assessment while the patient is traveling to imaging, having the tPA mixed by the time the non-contrast CT has occurred, and administrating tPA after the non-contrast CT but before the CTA. It is important to note that the model assumes $100 \%$ of stroke protocol compliance and implementation of test scenarios. For example, when testing the process change of taking a patient to imaging on the EMS stretcher, personnel dependent variation in compliance or variation based on patient factors may dictate the need to move the patient to an ED bay for stabilization prior to imaging was not incorporated. The main limitation of the DES model was the lack of available data detailing activity durations. The model would benefit by employing a time study and replacing estimated durations with collected data for improved accuracy. A second limitation is the use of assumptions and simplifications. The scope of the study was limited by focusing on thrombolysis and not including EVT. It is recognized that rural hospitals face additional challenges, such as arranging transfer of patients for EVT, deserving further study.

\section{CONCLUSION}

The conceptual framework developed in this study defines treatment pathways for AIS patients receiving thrombolysis treatment in urban or rural hospitals. The detail of the framework aims to reduce the gap between clinical knowledge and modeler perceptions regarding the logistics of the intrahospital aspect of the thrombolysis process. Several process improvements were shown to have a positive impact on median DNT and $90^{\text {th }}$ percentile DNT in urban and rural settings. Our modeling shows that of the three included process changes, the most significant median DNT improvement at rural hospitals resulted from ensuring patients arriving via EMS remain on the EMS stretcher to imaging, while urban sites benefit greater from administering tPA in the imaging area. Reducing the durations of activities found on the critical path will result in further DNT improvements. The lowest median DNTs and $90^{\text {th }}$ percentile DNTs results were achieved by combining all three process changes and two reduction in activity durations for all sites. Significant DNT improvements are achievable in urban and rural settings by combining process changes and reducing activity durations. Based on the results of this study, similar sites can feel confident in achieving desired outcomes when implementing these process improvements. Discrete-event simulation modeling of acute stroke processes can be expanded to develop more accurate models to reveal the ideal processes to achieve the most efficient treatment for patients.

\section{DATA AVAILABILITY STATEMENT}

The original contributions presented in the study are included in the article/Supplementary Material, further inquiries can be directed to the corresponding author/s.

\section{AUTHOR CONTRIBUTIONS}

TB: study design, DES modeling, data analysis, preparation of figures and tables, preparation of the first and revised drafts of the manuscript, as well as final editing, and formatting. DV: revision of manuscript for intellectual content. JB: input into the following areas: data analysis, model verification, sensitivity analysis, statistical analysis, as well as revision of manuscript for intellectual content. NK: study design, input into data analysis, editing, formatting, and revision of manuscript for intellectual content. All authors contributed to the article and approved the submitted version. 


\section{FUNDING}

This work was funded by Canadian Institutes of Health Research (CIHR) Project Grant (PJT-169124) held by NK.

\section{REFERENCES}

1. The National Institute of Neurological Disorders and Stroke rt-PA Stroke Study Group. Tissue plasminogen activator for acute ischemic stroke. N Engl J Med. (1995). 333:1581-7. doi: 10.1056/NEJM199512143332401

2. Berkhemer OA, Fransen PSS, Beumer D, van den Berg LA, Lingsma HF, Yoo AJ, et al. A randomized trial of intraarterial treatment for acute ischemic stroke. N Engl J Med. (2015) 372:11-20. doi: 10.1056/NEJMoa1411587

3. Jovin TG, Chamorro A, Cobo E, de Miquel MA, Molina CA, Rovira A, et al. Thrombectomy within 8 hours after symptom onset in ischemic stroke. N Engl J Med. (2015) 372:2296-306. doi: 10.1056/NEJMoa1503780

4. Saver JL, Goyal M, Bonafe A, Diener H-C, Levy EI, Pereira VM, et al. Stentretriever thrombectomy after intravenous t-PA vs. t-PA Alone in Stroke. $N$ Engl J Med. (2015) 372:2285-95. doi: 10.1056/NEJMoa1415061

5. Goyal M, Demchuk AM, Menon BK, Eesa M, Rempel JL, Thornton J, et al. Randomized assessment of rapid endovascular treatment of ischemic stroke. N Engl J Med. (2015) 372:1019-30. doi: 10.1056/NEJMoa1414905

6. Campbell BCV, Mitchell PJ, Kleinig TJ, Dewey HM, Churilov L, Yassi N, et al. Endovascular therapy for ischemic stroke with perfusion-imaging selection. $N$ Engl J Med. (2015) 372:1009-18. doi: 10.1056/NEJMoa1414792

7. Saver JL. Time is brain - quantified. Stroke. (2006) 37:263-6. doi: 10.1161/01.STR.0000196957.55928.ab

8. Lees KR, Bluhmki E, von Kummer R, Brott TG, Toni D, Grotta JC, et al. Time to treatment with intravenous alteplase and outcome in stroke: an updated pooled analysis of ECASS, ATLANTIS, NINDS, and EPITHET trials. Lancet. (2010) 375:1695-703. doi: 10.1016/S0140-6736(10)60491-6

9. Goyal M, Almekhlafi M, Dippel DW, Campbell BCV, Muir K, Demchuk AM, et al. Rapid alteplase administration improves functional outcomes in patients with stroke due to large vessel occlusions: meta-analysis of the noninterventional arm from the HERMES collaboration. Stroke. (2019) 50:645-51. doi: 10.1161/STROKEAHA.118.0 21840

10. Emberson J, Lees KR, Lyden P, Blackwell L, Albers G, Bluhmki E, et al. Effect of treatment delay, age, and stroke severity on the effects of intravenous thrombolysis with alteplase for acute ischaemic stroke: a meta-analysis of individual patient data from randomised trials. Lancet. (2014) 384:1929-35. doi: 10.1016/S0140-6736(14)60584-5

11. Kamal N, Jeerakathil T, Stang J, Liu M, Rogers E, Smith EE, et al. Provincial door-to-needle improvement initiative results in improved patient outcomes across an entire population. Stroke. (2020) 51:2339-46. doi: 10.1161/STROKEAHA.120.029734

12. Meretoja A, Strbian D, Mustanoja S, Tatlisumak T, Lindsberg PJ, Kaste M. Reducing in-hospital delay to 20 minutes in stroke thrombolysis. Neurology. (2012) 79:306-13. doi: 10.1212/WNL.0b013e31825d6011

13. Bulmer T, Volders D, Kamal N. Analysis of thrombolysis process for acute ischemic stroke in urban and rural hospitals in Nova Scotia Canada. Front Neurol. (2021) 12:1-15. doi: 10.3389/fneur.2021.645228

14. Kamal N, Smith EE, Jeerakathil T, Hill MD. Thrombolysis: improving doorto-needle times for ischemic stroke treatment - a narrative review. Int J Stroke. (2017) 13:268-76. doi: 10.1177/1747493017743060

15. Saghafian S, Austin G, Traub SJ. Operations research/management contributions to emergency department patient flow optimization: review and research prospects. IIE Trans Healthc Syst Eng. (2015) 5:101-23. doi: 10.1080/19488300.2015.1017676

16. Churilov L, Fridriksdottir A, Keshtkaran M, Mosley I, Flitman A, Dewey HM. Decision support in pre-hospital stroke care operations: a case of using simulation to improve eligibility of acute stroke patients for thrombolysis treatment. Comput Oper Res. (2013) 40:2208-18. doi: $10.1016 /$ j.cor.2012.06.012

\section{SUPPLEMENTARY MATERIAL}

The Supplementary Material for this article can be found online at: https://www.frontiersin.org/articles/10.3389/fneur. 2021.746404/full\#supplementary-material

17. Bogle BM, Asimos AW, Rosamond WD. Regional evaluation of the severity-based stroke triage algorithm for emergency medical services using discrete event simulation. Stroke. (2017) 48:2827-35. doi: 10.1161/STROKEAHA.117.017905

18. Stahl JE, Furie KL, Gleason S, Gazelle GS. Stroke: effect of implementing an evaluation and treatment protocol compliant with NINDS recommendations. Radiology. (2003) 228:659-68. doi: 10.1148/radiol.2283021557

19. Komenda I, Knight V, Williams HM. Compliance with national guidelines for stroke in radiology. Oper Res Heal Care. (2015) 6:33-9. doi: 10.1016/j.orhc.2015.09.001

20. Uzun Jacobson E, Bayer S, Barlow J, Dennis M, MacLeod MJ. The scope for improvement in hyper-acute stroke care in Scotland. Oper Res Heal Care. (2015) 6:50-60. doi: 10.1016/j.orhc.2015.09.003

21. Huang S, Maingard J, Kok HK, Barras CD, Thijs V, Chandra R V., et al. Optimizing resources for endovascular clot retrieval for acute ischemic stroke, a discrete event simulation. Front Neurol. (2019). 10:653. doi: 10.3389/fneur.2019.00653

22. Monks T, Pitt M, Stein K, James M. Maximizing the population benefit from thrombolysis in acute ischemic stroke: a modeling study of in-hospital delays. Stroke. (2012) 43:2706-11. doi: 10.1161/STROKEAHA.112.663187

23. Lahr MMH, van der Zee D-J, Luijckx G-J, Vroomen PCAJ, Buskens E. A simulation-based approach for improving utilization of thrombolysis in acute brain infarction. Med Care. (2013) 51:1101-5. doi: 10.1097/MLR.0b013e3182a3e505

24. Lahr MMH, Luijckx G-J, Vroomen PCAJ, van der Zee D-J, Buskens E. The chain of care enabling tPA treatment in acute ischemic stroke: a comprehensive review of organisational models. J Neurol. (2013) 260:960-8. doi: 10.1007/s00415-012-6647-7

25. Lahr MMH, van der Zee D-J, Luijckx G-J, Vroomen PCAJ, Buskens E. Centralising and optimising decentralised stroke care systems: a simulation study on short-term costs and effects. BMC Med Res Methodol. (2017) 17:1-12. doi: 10.1186/s12874-016-0275-3

26. Bayer S, Petsoulas C, Cox B, Honeyman A, Barlow J. Facilitating stroke care planning through simulation modelling. Health Inform J. (2010) 16:129-43. doi: $10.1177 / 1460458209361142$

27. Lahr MMH, van der Zee D-J, Vroomen PCAJ, Luijckx G-J, Buskens E. Thrombolysis in acute ischemic stroke: a simulation study to improve preand in-hospital delays in community hospitals. PLoS ONE. (2013) 8:e79049. doi: 10.1371/journal.pone.0079049

28. Lahr MMH, van der Zee D-J, Luijckx G-J, Buskens E. Optimising acute stroke care organisation: a simulation study to assess the potential to increase intravenous thrombolysis rates and patient gains. BMJ Open. (2020) 10:e032780. doi: 10.1136/bmjopen-2019-032780

29. Lahr MMH, Maas WJ, van der Zee D-J, Uyttenboogaart M, Buskens E. Rationale and design for studying organisation of care for intra-arterial thrombectomy in the Netherlands: simulation modelling study. BMJ Open. (2020) 10:e032754. doi: 10.1136/bmjopen-2019-032754

30. Chemweno P, Thijs V, Pintelon L, Van Horenbeek A. Discrete event simulation case study: diagnostic path for stroke patients in a stroke unit. Simul Model Pract Theory. (2014) 48:45-57. doi: 10.1016/j.simpat.2014.07.006

31. McMeekin P, Flynn D, Allen M, Coughlan D, Ford GA, Lumley H, et al. Estimating the effectiveness and cost-effectiveness of establishing additional endovascular thrombectomy stroke centres in England: a discrete event simulation. BMC Health Serv Res. (2019) 19:821. doi: 10.1186/s12913-019-4678-9

32. Hoffmeister L, Lavados PM, Mar J, Comas M, Arrospide A, Castells X. Minimum intravenous thrombolysis utilization rates in acute ischemic stroke to achieve population effects on disability: a discrete-event simulation model. J Neurol Sci. (2016) 365:59-64. doi: 10.1016/j.jns.2016.04.005 
33. Monks T, van der Zee D-J, Lahr MMH, Allen M, Pearn K, James MA, et al. A framework to accelerate simulation studies of hyperacute stroke systems. Oper Res Heal Care. (2017) 15:57-67. doi: 10.1016/j.orhc.2017.09.002

34. Monks T, Currie CSM, Onggo BS, Robinson S, Kunc M, Taylor SJE. Strengthening the reporting of empirical simulation studies: introducing the STRESS guidelines. J Simul. (2019) 13:55-67. doi: 10.1080/17477778.2018.1442155

35. Xian Y, Xu H, Lytle B, Blevins J, Peterson ED, Hernandez AF, et al. Use of strategies to improve door-to-needle times with tissue-type plasminogen activator in acute ischemic stroke in clinical practice: findings from target: stroke. Circ Cardiovasc Qual Outcomes. (2017) 10:1-8. doi: 10.1161/CIRCOUTCOMES.116.003227

36. Meretoja A, Weir L, Ugalde M, Yassi N, Yan B, Hand P, et al. Helsinki model cut stroke thrombolysis delays to 25 minutes in melbourne in only 4 months. Neurology. (2013) 81:1071-6. doi: 10.1212/WNL.0b013e3182a4a4d2

37. Thortveit ET, Bøe MG, Ljøstad U, Mygland A, Tveiten A. Organizational changes aiming to reduce IV tPA door-to-needle time. Acta Neurol Scand. (2014) 130:248-52. doi: 10.1111/ane.12204

38. Van Schaik SM, Van der Veen B, Van den Berg-Vos RM, Weinstein HC, Bosboom WMJ. Achieving a door-to-needle time of 25 minutes in thrombolysis for acute ischemic stroke: a quality improvement project. J Stroke Cerebrovasc Dis. (2014) 23:2900-6. doi: 10.1016/j.jstrokecerebrovasdis.2014.07.025

39. Busby L, Owada K, Dhungana S, Zimmermann S, Coppola V, Ruban R, et al. CODE FAST: a quality improvement initiative to reduce door-to-needle times. J Neurointerv Surg. (2016) 8:661-4. doi: 10.1136/neurintsurg-2015-011806

40. Kamal N, Holodinsky JK, Stephenson C, Kashayp D, Demchuk AM, Hill $\mathrm{MD}$, et al. Improving door-to-needle times for acute ischemic stroke: effect of rapid patient registration, moving directly to computed tomography, and giving alteplase at the computed tomography scanner. Circ Cardiovasc Qual Outcomes. (2017) 10:1-8. doi: 10.1161/CIRCOUTCOMES.116.003242

41. Zinkstok SM, Beenen LF, Luitse JS, Majoie CB, Nederkoorn PJ, Roos YB. Thrombolysis in stroke within 30 minutes: results of the acute brain care intervention study. PLoS ONE. (2016) 11:1-15. doi: 10.1371/journal.pone.0166668

42. Ford AL, Williams JA, Spencer M, McCammon C, Khoury N, Sampson TR, et al. Reducing door-to-needle times using toyota's lean manufacturing principles and value stream analysis. Stroke. (2012) 43:3395-8. doi: 10.1161/STROKEAHA.112.670687
43. Holodinsky JK, Kamal N, Wilson AT, Hill MD, Goyal M. Workflow in acute stroke: what is the 90th percentile? Stroke. (2017) 48:808-12. doi: 10.1161/STROKEAHA.116.015951

44. Boulanger JM, Lindsay MP, Gubitz G, Smith EE, Stotts G, Foley N, et al. Canadian stroke best practice recommendations for acute stroke management: prehospital, emergency department, and acute inpatient stroke care, 6th edition, update 2018. Int J Stroke. (2018) 13:949-84. doi: $10.1177 / 1747493018786616$

45. Stellman R, Redfern A, Lahri S, Esterhuizen T, Cheema B. How much time do doctors spend providing care to each child in the ED? A time and motion study. Emerg Med J. (2021). 2021:emermed-2019-208903. doi: 10.1136/emermed-2019-2 08903

46. Schneider A, Williams DJ, Kalynych C, Wehler M, Weigl M. Physicians' and nurses' work time allocation and workflow interruptions in emergency departments: a comparative time-motion study across two countries. Emerg Med J. (2021) 38:263-8. doi: 10.1136/emermed-2019-208508

Conflict of Interest: NK: Founder and part equity owner of DESTINE Health. Principal Investigator for CIHR grant that funded this work.

The remaining authors declare that the research was conducted in the absence of any commercial or financial relationships that could be construed as a potential conflict of interest.

Publisher's Note: All claims expressed in this article are solely those of the authors and do not necessarily represent those of their affiliated organizations, or those of the publisher, the editors and the reviewers. Any product that may be evaluated in this article, or claim that may be made by its manufacturer, is not guaranteed or endorsed by the publisher.

Copyright (C) 2021 Bulmer, Volders, Blake and Kamal. This is an open-access article distributed under the terms of the Creative Commons Attribution License (CC BY). The use, distribution or reproduction in other forums is permitted, provided the original author(s) and the copyright owner(s) are credited and that the original publication in this journal is cited, in accordance with accepted academic practice. No use, distribution or reproduction is permitted which does not comply with these terms. 